\title{
Letter to the Editor concerning "Is L5-S1 motion segment different from the rest? A radiographic kinematic assessment of 72 patients with chronic low back pain" by AB Sabnis et al. (Eur Spine J; 27(5):1127-1135)
}

\author{
Miao $\mathrm{Yu}^{1} \cdot \mathrm{Xu} \mathrm{Wang}^{2,3}$
}

Received: 30 May 2018 / Accepted: 17 June 2018 / Published online: 21 June 2018

(c) Springer-Verlag GmbH Germany, part of Springer Nature 2018

\section{Dear Editor,}

Recently, we read with great interest the well-written article entitled "Is L5-S1 motion segment different from the rest? A radiographic kinematic assessment of 72 patients with chronic low back pain" [1]. The authors concluded that L5-S1 showed different motion and degenerative characteristics compared with the rest levels of the lumbar spine. This is interesting since it may provide important evidence for assessing segmental range of motion (ROM) without L5-S1 included to increase the data homogeneity.

We appreciate the tremendous work by the authors for discovering the unique characteristics in the lumbar spine. However, there are several issues on the assessment of disc degeneration and spinal kinematics we would like to discuss with the authors.

First, it might be better if the weight-bearing magnetic resonance imaging (MRI) of the lumbar spine was applied because the disc height will be altered in the supine position compared with it in the standing position. Thus, the association between disc degeneration in the supine position and segmental ROM in the standing radiographs may alter when the two (disc degeneration and ROM) were assessed in the standing position.

Xu Wang

x.wang2013@ hotmail.com

1 Department of Ophthalmology, The Second Hospital of Jilin University, Changchun, Jilin, China

2 Sensory-Motor Interaction, Department of Health Science and Technology, Faculty of Medicine, Aalborg University, Fredrik Bajers Vej 7E-2, 9220 Aalborg, Denmark

3 Department of Orthopedics, The Second Hospital of Jilin University, Changchun, China
Second, the height of the disc scored referring to the adjacent disc may be a problem as the adjacent disc in patients with chronic low back pain (for instance 3 years) almost lost its normal anatomy compared to the healthy controls. However, this issue has already been addressed in the limitations of the study.

Third, the segmental ROM of the spine was reported to be affected by the alignment [2]. It is obvious that the routine MRI shows a relatively "straight" lumbar spine and this may partly be due to the supine position, which means it cannot reflect the actual alignment of the lumbar spine. The effects of different types of lumbar alignment (kyphosis, lordosis, straight and reverse) on segmental ROMs need to be investigated before the evidence is further applied in both clinical and scientific practices.

We again appreciate the work by the authors; it pointed to the future studies to exclude L5-S1 when analysing the lumbar spine kinematics and disc degeneration to increase data/ sample homogeneity. However, several issues mentioned above need to be considered prior to its further application.

\section{Compliance with ethical standards}

Conflict of interest The authors declare that they have no conflict of interest.

\section{References}

1. Sabnis AB, Chamoli U, Diwan AD (2018) Is L5-S1 motion segment different from the rest? A radiographic kinematic assessment of 72 patients with chronic low back pain. Eur Spine J 27(5):1127-1135

2. Sessumpun K, Paholpak P, Hindoyan KN et al (2018) Characteristics of cervical spine motion in different types of cervical alignment: kinematic MRI study. Clin spine Surg 31:E239-E244. https://doi.org/10.1097/BSD.0000000000000605 